
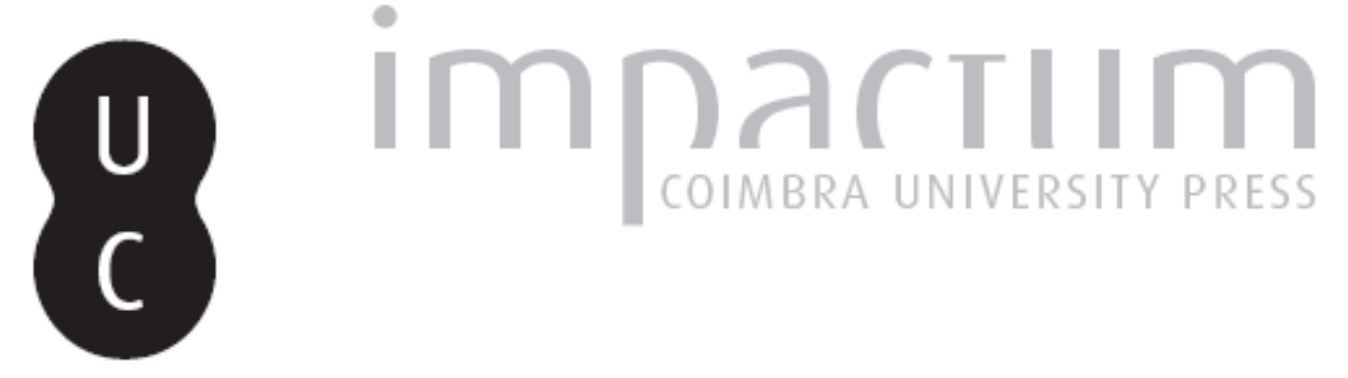

\title{
[Recensão a] Lidio Gasperini, Iscrizioni latine rupestri nel Lazio. I - Etruria Meridionale. "Richerche sul Lazio"1
}
Autor(es):
Simula, Manuela

Publicado por: Imprensa da Universidade de Coimbra

URL

persistente:

URI:http://hdl.handle.net/10316.2/45400

DOI:

DOI:https://dx.doi.org/10.14195/1647-8657_38_15

Accessed : $\quad$ 26-Apr-2023 14:58:15

A navegação consulta e descarregamento dos títulos inseridos nas Bibliotecas Digitais UC Digitalis, UC Pombalina e UC Impactum, pressupõem a aceitação plena e sem reservas dos Termos e Condições de Uso destas Bibliotecas Digitais, disponíveis em https://digitalis.uc.pt/pt-pt/termos.

Conforme exposto nos referidos Termos e Condições de Uso, o descarregamento de títulos de acesso restrito requer uma licença válida de autorização devendo o utilizador aceder ao(s) documento(s) a partir de um endereço de IP da instituição detentora da supramencionada licença.

Ao utilizador é apenas permitido o descarregamento para uso pessoal, pelo que o emprego do(s) título(s) descarregado(s) para outro fim, designadamente comercial, carece de autorização do respetivo autor ou editor da obra.

Na medida em que todas as obras da UC Digitalis se encontram protegidas pelo Código do Direito de Autor e Direitos Conexos e demais legislação aplicável, toda a cópia, parcial ou total, deste documento, nos casos em que é legalmente admitida, deverá conter ou fazer-se acompanhar por este aviso.

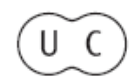


UNIVERSIDADE DE COIMBRA

FACULDADE DE LETRAS

\section{CONIMBRIGA}

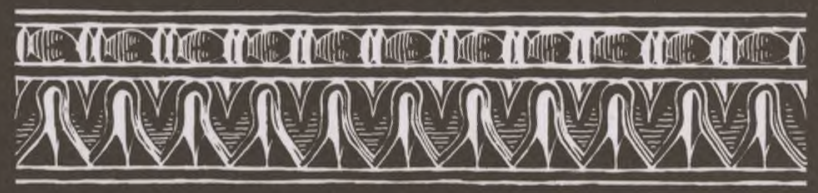

VOLUME XXXVIII - 1999 
do Museu de Timgad e na p. 47, com a personificação de um rio de uma residência de Annaba. Adiante, na p. 53, não vêm ilustrados outros dois mosaicos também expostos no Museu de Oran. Nap. 101, pela primeira vez, a A. explica a ausência de ilustração do mosaico de Vénus e das Nereides do triclinium da Casa de Vénus em Annaba, por este se encontrar coberto. Na p. 126, também nos privaram do mosaico de Cila, do Museu de Hippone e, na p. 147, foi a vez do pescador de dourada de Djemila.

- É frequente urna certa desorganização na apresentação das legendas.

De facto, são incluídas ampliações de pormenores de mosaicos cuja identificação é omitida ou deslocada para outra página. Verificámo-lo nas pp. 22-23, 50-53, 55-59, 86-87, 152-153, 166-167 el76.

- Por falta de correcta revisão da obra, duas fotografias estão invertidas: a ampliação da Nereide situada mais à esquerda do mosaico do Museu de Lambèse (p. 59) e o mosaico de Ulisses e as Sereias de Cherchel (pp. 118-119).

- Apesar de nos termos já pronunciado quanto à qualidade da maior parte das fotografias, não podemos deixar de lamentar a má qualidade da do mosaico marinho com máscaras de Oceano do Museu de Hippone (Annaba) que invalida qualquer tipo de leitura (pp. 26-27).

Melhor síntese final não poderia ter sido feita pela A.: a água é, simultaneamente, fonte de vida, regeneradora e alimentadora, meio de comunicação mas também lugar de poderes maléficos. Esta relação que o romano manteve com o meio marinho, ao mesmo tempo frutífera e aterradora, foi de tal forma interiorizada que as suas manifestações não se restringem às zonas litorais, mas foram transportadas para regiões interiores, onde já não é o mar que apela, mas sim as fontes e os rios, tão elementares para a vida humana. As províncias do norte de África, mais do que qualquer outra parte do Império, receberam do Mar grande parte da sua riqueza económica e da sua cultura que, através dele, souberam, sabiamente, devolver aos outros. O mare nostrum, centro nevrálgico da economia romana, ligando os dois Impérios, ocidental e oriental, foi perdendo o seu papel ao longo da História, em favor de outros mares, contudo, para a memória do Homem, ele perdurará como um importante pòlo cultural e como a eterna morada dos deuses do Olimpo.

CRISTINA DE OLIVEIRA

LIDIO GASPERINI, Iscrizioni latine rupestri nel Lazio. I - Etruria Meridionale. "Richerche sul Lazio" 1. Roma, 1989, 156 p. + tav. XXXII.

Pubblicato nel 1989, il volume del Gasperini rende conto dello studio condotto dal Dipartimento di Storia dell II Università di Roma su una particolare tipologia di manufatto epigrafico: le iscrizioni rupestri latine, limitatamente all'Alto Lazio.

Conimbriga, 38 (1999), p. 225-246 
L'area, non molto vasta, pure fornisce un numero considerevole di testimonianze, perlomeno pari al totale delle presenze epigrafiche rupestri nel resto dell'Italia; motivo sarebbe non una eccezionale ricchezza litica della regione, quanto la familiarità della sua popolazione con l'elemento roccia. Questo supporto epigrafico, evidentemente non mobile, fa sì che le iscrizioni stiano lì dove sono state pensate e realizzate (quando non sopraggiungono smottamenti e sbancamenti); se il fatto che siano in situ permette la conoscenza del preciso contesto archeologico, nel contempo pone diversi problemi di accessibiltà e reperimento del manufatto. Bastino come esempio le iscrizioni presentate con il numero $\mathrm{E}_{28}$ ed $\mathrm{E}_{32}$ : la prima si trova in una zona boschiva particolarmente intricata ed è oggi ricoperta da muschi e licheni; l'altra, facente parte di una tagliata stradale, è precipitata e costituisce il soffitto di un recente camminamento.

Nel volume vengono analizzate 38 iscrizioni rupestri, di cui due inedite $\left(\mathrm{E}_{1}\right.$ ed $\left.\mathrm{E}_{29}\right)$ le prime dieci in provincia di Roma, le altre in quella di Viterbo.

Due i gruppi principali: le viarie-confinarie (in tutto 18) e le sepolcrali (16), cui si aggiungono una acclamatoria, due sacre ed una agraria. Cronologicamente si collocono tra il II a. C. e il II d. C. Di queste iscrizioni la prima sezione presenta il panorama delle dislocazioni con riferimento alla Forma Italiae per 1'Etruria romana e alle Tavolette I. G. M. per i comprensori moderni (in tutto dieci comuni) con una descrizione puntuale della ubicazione e della morfologia del sito, l'accessibilità e la natura litologica del supporto epigrafico.

Sono informazioni utili per accedere direttamente agli originali, per stabilire la pertinenza ai singoli comuni del patrimonio archeologico da proteggere, per relazionare la struttura petrográfica dell'iscrizione con la sua tipologia: se le sepolcrali per esempio le troviamo in tufi litoidi, in quanto più resistenti, quelle viarie compaiono generalmente in tufi pomicei, più adatti, perchè più teneri, all'escavazione di tagliate viarie.

La seconda sezione è costituita dal catalogo delle iscrizioni presentate con il criterio topografico del C. I. L., in senso antiorario dalla costa all'interno.

Le prime sette sono iscrizioni confinarie che colpiscono per la loro ermeticità: su massi trachitici, diversi per forma e dimensioni, compaiono cifre numerali accompagnate da segmenti di retta ad innesto ed orientamento specifici di cui il Gasperini fornisce misure ed angolazioni. Si tratterebbe o di termini rupestri che limitano un'area sacra, oppure di scritte terminali tra territori pubblici e privati. In ogni caso, le linee indicherebbero il punto di passaggio del confine; il punto in cui esso muta o anche rincontro di due linee di confine. Il terreno impervio farebbe pensare a separazioni tra terreni pubblici e privati, ma niente è stato verificato sul campo. Se normalmente il terminus latino, quando in situ, fornisce informazioni sull'organizzazione politico-amministrativa del territorio, sull'onomastica, sulle popolazioni e sulla loro dislocazione, qui tutto questo manca; la Sententia Minuciorum del 117 a. C. che è riportata come confronto, dà l'idea di quelle parole che gli artefici delle scritte consideravono superflue nel rapportarsi alla concretezza di un possedimento terriero.

Viarie sono invece le iscrizioni $\mathrm{E}_{13} ; \mathrm{E}_{15}$; il gruppo dalla $\mathrm{E}_{30}$ alla $\mathrm{E}_{38}$. La 
prima desta interesse dal punto di vista linguistico in quanto testo latino-falisco: falisci sono i numeri che gli scavatori della tagliata hanno inciso a ricordo del compito assegnatogli (come resistere al desiderio tutto umano di lasciare un segno di sè?! !); latino è invece il testo in cui stupisce la presenza del termine furca che, se frequente nella toponomastica della zona, lo è molto meno nelle epigrafi ad indicare uno stretto passaggio.

Il gruppo dalla $\mathbf{E}_{\mathbf{3 0}}$ alla $\mathbf{E}_{\mathbf{3 8}}$ fornisce invece informazioni sulla rete stradale della regio VII, dati utili per l'antroponomastica, testimonianze di opere di evergetismo.

L'iscrizione $\mathbf{E}_{33}$, per esempio, ricorda un certo Gaio Clodio Talpio che a sue spese fece fare un tratto di strada di 40 passi. È l'atto evergetico di un liberto legato alla famiglia Clodia che vuole partecipare in maniera importante $(59,2 \mathrm{~m})$ alla costruzione dell'iter publicum dedicato alla gens che lo ha manomesso. Lontano dal caput viae e dal Forum Clodii si concedeva eternità a quei privati cittadini che sostenessero e accelerassero il completamento della via.

Fa pendant con questa iscrizione la $\mathbf{E}_{\mathbf{3 0}}$ in cui un gruppo di liberti rivendica la parte di strada che ha fatto costruire; si tratta di un iter privatum, ma l'aspettativa degli evergeti è la stessa: un ricordo duraturo per aver contribuito ad un'opera non pubblica ma ugualmente importante per le popolazioni locali.

Lo dimostra l'iscrizione $\mathbf{E}_{\mathbf{3 2}}$ : virum bo/num, qui ha/nc cons/iliavit! un'acclamazione all'anonimo costruttore la cui benemerenza è celebrata continuamente da chi passa in quella strada; una tappa obbligata per tuttti i viatores che leggendo l'iscrizione perpetuono la donazione del tal personaggio tanto noto da non sentire la necessità di manifestarsi.

Gruppo rilevante è quello delle iscrizioni sepolcrali.

Due sono tituli pedaturae: la $\mathbf{E}_{\mathbf{n}}$, pertinente ad un colombario e la $\mathbf{E}_{\mathbf{1 2}}$, di un ipogeo sepolcrale, di cui poco si sa per l'assenza di foto ed apografi.

Quattro sono invece le iscrizioni funerarie che fanno riferimento a sepolture terragnee collocate ai piedi di un masso iscritto: $\mathbf{E}_{\mathbf{8}} ; \mathbf{E} \mathbf{E}_{\mathbf{7}} ; \mathbf{E}_{\mathbf{2 7}} ; \mathbf{E}_{\mathbf{2 8}}$. Maggiore attenzione richiedono a mio parere la $\mathbf{E}_{\mathbf{1 7}}$ e la $\mathbf{E}_{\mathbf{2 8}}$.

Nel primo caso l'interesse è onomastico: trattasi probabilmente di una coppia di sposi. Lui, M. Avilius Celer, ingenuus, è membro di una gens già attestata in Etruria, è iscritto all tribù Collina e per questo probabilmente originario délV Urbe; lei, liberta, si distingue per un nome servile, Herennia Guidila (gocciolina), tanto raro quanto prezioso nella sua derivazione naturale.

Per la $\mathbf{E}_{\mathbf{2 8}}$ la particolarità risiede invece nel fatto che il monumento cui appartiene, il sepolcro dei Vibii, è uno dei primi esempi di fossa «a logette» dell'Etruria alto-imperiale. Alla prima fase in cui la corrispondenza tra cavi sepolcrali (fossa e loculo) e defunti citati è perfetta, segue una seconda che nel monumento è solo epigrafica.

Un'analisi paleografica permette di distinguere Vordinatio ineccepibile, l'incizione accurata, le lettere calibrate e le interpunzioni di fattura officinale dei primi due personaggi, Q. Vibius Rufus e Vibia Arbuscula, dalla realizzazione artigianale del nome del liberto $Q$. Vibius Faustus. 
Altra iscrizione importante è la $\mathrm{E}_{22}$, l'unica leggibile delle tre presenti nel macigno di Anicio.

È rilevante perchè il citato C. Anicius è quattuorvir Statoni: per la prima volta Statonia può identificarsi come municipium. Questo significa che il suo agro era independente, che gli Statonienses erano una delle popolazioni della Regio VII e che erano governati da magistrati loro propri.

Non solo una roccia, ma un documento sull'evoluzione dello status di una città.

Tra le iscrizioni sepolcrali ricorderei ancora la $\mathrm{E}_{29}$ una delle due inedite, che rende conto del grande dramma della mortalità infantile; nella semplice e cruda frase Pueri hic co/nditi accomuna il triste destino di tanti anonimi bambini morti.

Le iscrizioni sacre sono soltanto due, pertinenti ad altretanti santuari rupestri: l'Altarone di Montevirginio $\left(\mathrm{E}_{9}\right)$ e l'altare dell'Arcella di Campina $\left(\mathrm{E}_{16}\right)$; geograficamente distanti, appartengono allo stesso panorama cultuale e religioso.

Entrambe le iscrizioni si riferiscono ad una Bona Dea, divinità ctonia e guaritrice, la cui forza le popolazioni di Forum Clodii da una parte e del Monte Cimino dall'altra vedevano emergere nei numerosi saxa vulcanici lì presenti, come anche nei fenomeni eruttivi che interessano entrambi i comprensori. Nel caso dell'Altarone di Montevirginio, la dea non è indicata esplicitamente, ma è normale che un monumento epigrafico posto all'interno del templum di una dea nota, o in ambiti santuariali monoculturali, non presenti il teonimo.

Nell'Altare dell'Arcella invece la Bona Dea (ricordata con la formula Bona Bonodiana Castrensis) è associata ad un'altra divinità, dal carattere ctonio anche lei: la dea Valetudo; questa sarebbe il nume specifico del santuario delle acque dell'Arcella, quella il nume tutelare di tutto il sito montano. Se esistono nel mondo romano manufatti rupestri che si riconducono a contesti sacrali, pochi sono legati a culti idrici (un esempio anche in Portogallo, a Bracara Augusta). Queste iscrizioni danno conto dell'acculturazione delle popolazioni montane, del grado di penetrazione dell'elemento romano, dei movimenti popolazionali. La Pacilia Primitiva che si rivolge alla dea Bonodiana Castrensis, oriunda di Roma, £a portato fuori da Würbe l'epiteto con cui si soleva rivolgere alla divinità a lei cara. Questi testi ci mostrano poi anche fenomeni di sincretismo: se la Bona Dea è l'antica Suri etrusca, la Valetudo riprenderebbe il culto della dea Salus.

Chiudo con un testo, $\mathrm{E}_{14}$, interessante perchè riporta la realizzazione di prata privati con opere di bonifica agraria, in genere non comuni.

Il merito di questo testo risiede principalmente nell'aver dato luce ad un patrimonio epigrafico che tende sempre a rimanere nell'ombra. L'Italia non possiede un censimento generale delle sue iscrizioni latine rupestri e questa è una grave mancanza sopratutto nel momento in cui si voglia promuoverne la tutela, o, ancora di più, affiancare all'analisi epigrafica un'indagine archeologica.

$\mathrm{Nel}$ caso delle iscrizioni confinarie per esempio, verificare sul terreno quanto si è ipotizzato avrebbe dato verosimiglianza e solidità alle interpretazioni proposte: 
sono manufatti fissi, in luoghi perlopiù inaccessibili, da cui è escluso il grande pubblico, non possono nè esseri visti in un museo, nè adeguatamente protetti.

Una pubblicazione come questa, che negli intenti dell'autore è la prima di una serie, può motivare iniziative similari. Solo un'indagine archeologica può per esempio illuminare sulle testimonianze che, in termini di cultura materiale, hanno lasciato i frequentatori delle aree sacre alla Bona Dea.

La bibliografia proposta va degli imprescindibili C. I. L.; C. I. E.; L'Année Epigraphique..., a testi legati alla situazione epigrafica, religiosa, sociale, giuridica dell'Etruria romana, ma avrei voluto per esempio trovare l'indicazione di trattazioni generali sulle epigrafi rupestri, utili per un profano, anche in riferimento a zone diverse dall'Alto Lazio.

Esaustivo e di facile consultazione l'indice epigrafico, mentre meno ho apprezzato le tavole fotografiche, la cui riuscita dipende inevitabilmente dal grado di accessibilità di queste iscrizioni.

La pubblicazione ha suscitato il mio interesse sia per l'argomento trattato, perchè non molto sopevo sulle iscrizioni rupestri, sia perchè ho potuto vedere come si procede tecnicamente nello studio e nella edizione di un ritrovamento epigrafico.

Ho visto corno è concretamente possibile andare oltre il testo e ricostruire le circostanze che hanno portato alla composizione di quel messaggio, immaginare i contesti sociali, culturali, economici che l'hanno prodotto. Se le minuziose descrizioni dei manufatti, i precisi commentari paleografici, la bibliografia, le varianti di lettura proposte possono non incuriosire, a volte stancare il lettore inesperto, presentare una umanità più piccola rispetto a quella delle grandi cariche dello Stato, quella più vera e più autentica del $M$. Lucilio dell'iscrizione $\mathrm{E}_{18}$ che incide con le sue mani 1'epitafio della madre, può far guadagnare ad opere di questo genere un pubblico più ampio, ispirandogli sia il rispetto per il patrimonio archeologico che interventi di evergetismo per la sua valorizzazione.

ManUela Simula

Conimbriga, 38 (1999), p. 225-246 